INPLASY

PROTOCOL

To cite: Chen et al. Triple

Combination of

Hydrocortisone, Ascorbic Acid, and Thiamine (HAT) for Sepsis/

Septic Shock: Meta-analysis and Trial Sequential Analysis. Inplasy protocol 202090097. doi:

10.37766/inplasy2020.9.0097

Received: 27 September 2020

Published: 27 September 2020

Corresponding author:

Yen Ta Huang

uncleda.huang@gmail.com

Author Affiliation:

Division of Experimental

Surgery, Department of

Surgery, Buddhist Tzu Chi

General Hospital, Hualien,

Taiwan

Support: N/A.

Review Stage at time of this submission: Data analysis.

Conflicts of interest: N/A.

\section{Triple Combination of Hydrocortisone, Ascorbic Acid, and Thiamine (HAT) for Sepsis/Septic Shock: Meta-analysis and Trial Sequential Analysis}

Chen, $\mathrm{CH}^{1}$; Huang, EP2; d'Etienne J33 Chou, EH4ㄴ Liu, YH5; Huang, YS6; Chang, JH7; Lai, PC8; Huang, YT9.

Review question / Objective: Does combining hydrocortisone, ascorbic acid, and thiamine have benefits of mortality, organ dysfunction, and cost-effectiveness on sepsis/septic shock patient?

Condition being studied: Several clinical trials and studies were performed to verify its effectiveness but showed divergence. The first systematic review with meta-analysis was published in Critical Care this year. The major difference from this prior article and our proposal is that Rui Shi and Hongtao Tie's study is a research letter. They did not present the flow chart of searching, the assessment of risk of bias, and the certainty of evidence.

INPLASY registration number: This protocol was registered with the International Platform of Registered Systematic Review and Meta-Analysis Protocols (INPLASY) on 27 September 2020 and was last updated on 27 September 2020 (registration number INPLASY202090097).

\section{INTRODUCTION}

Review question / Objective: Does combining hydrocortisone, ascorbic acid, and thiamine have benefits of mortality, organ dysfunction, and cost-effectiveness on sepsis/septic shock patient?
Rationale: The combination of hydrocortisone, ascorbic acid (vitamin C), and thiamine (vitamin B1), also called the HAT triple therapy, had been suggested in the treatment of sepsis and septic shock 
because of its anti-inflammatory and antioxidative features.

Condition being studied: Several clinical trials and studies were performed to verify its effectiveness but showed divergence. The first systematic review with metaanalysis was published in Critical Care this year. The major difference from this prior article and our proposal is that Rui Shi and Hongtao Tie's study is a research letter. They did not present the flow chart of searching, the assessment of risk of bias, and the certainty of evidence.

\section{METHODS}

Search strategy: Keywords using free texts and medical subject headings for search included "hydrocortisone," "ascorbic acid" or "vitamin C," "thiamine" or "vitamin B1," and "sepsis" or "septic shock.".

Participant or population: Sepsis/septic shock patients.

Intervention: HAT triple combination.

\section{Comparator: No HAT triple combination.}

Study designs to be included: The inclusion criteria were: 1) randomized controlled trials (RCTs), cohort, or case-control studies that involved patients with severe sepsis or septic shock; 2) intervention was HAT triple therapy; and 3) control group was patients treated with standard therapy, such as broad-spectrum antibiotics, fluid resuscitation, and vasopressors with or without hydrocortisone.

Eligibility criteria: The exclusion criteria were studies that did not meet the inclusion criteria; reviews, case reports, or case series; and those with no relevant data for extraction.

Information sources: Two independent investigators systematically searched PubMed, Web of Science, Embase, Airiti Library, and Cochrane Library from inception to August 31, 2020 without language limitation to enroll adequate pertinent studies.
Main outcome(s): The primary outcome was patient mortality, including ICU mortality, in-hospital mortality, and 28- or 30-day mortality.

Additional outcome(s): Secondary outcomes included the index of organ dysfunction and LOS. The index of organ dysfunction included the delta SOFA score at 72 hours after admission, durations of vasopressor and ventilator use, and the need for RRT. LOS included ICU LOS and hospital LOS.

Data management: Data were extracted from the eligible studies included by two authors, and a senior author finalized the data. The data extracted from the eligible studies included authors, publication year, study design, sex, age, numbers of cases and controls, initial SOFA score, treatment in intervention and control groups, and various outcomes of clinical interest.

Quality assessment / Risk of bias analysis: The risk of bias and internal validity were assessed by two senior authors independently using the "Risk-of-bias tool 2.0 (RoB 2.0)" for RCTs and the "Risk of Bias in Non-randomized Studies of Interventions (ROBINS-I) tool" developed by the Cochrane collaboration for nonrandomized studies. Divergences were resolved by consensus. The results of RoB 2.0 and ROBINS-I were drawn through the "Risk-of-Bias Visualization tool".

Strategy of data synthesis: Dichotomous outcomes were presented as risk ratio (RR), and continuous variables were presented as mean difference (MD), both with $95 \%$ confidence intervals (CIs). Continuous variables reported as median and interquartile range (IQR) was converted to mean and standard deviation (SD), respectively, using the equations: median = mean and $S D=I Q R / 1.35$ suggested by Handbook of Cochrane Review. Changes (mean and SD) of continuous variables in some endpoints were also calculated by the formula in Handbook of Cochrane Review. A random-effect model was used in the meta-analysis. We further performed subgroup analyses to distinguish the 
different pooled effects between RCTs and non-RCTs. Sensitivity analysis by metaregression of important endpoint was performed to explore covariate(s) which resulted in high heterogeneity of the metaIytic effects by the software OpenMetaAnalyst with random-effect model. Heterogeneities among studies were evaluated by the I square (I2) statistics. An 12 higher than $50 \%$ was considered substantially heterogeneous. Publication bias was assessed through visual inspection using funnel plots and quantified by the Doi plot (MetaXL version 5.3, EpiGear International Pvt., Ltd.). The meta-analysis was conducted using Review Manager (RevMan) version 5.3 (Copenhagen: The Nordic Cochrane Center, The Cochrane Collaboration, 2014). Hypothesis testing and heterogeneity testing were considered statistically significant if two-tailed $p<0.05$ and $p<$ 0.10 , respectively.

Subgroup analysis: RCTs and non-RCTs for the subgroup analysis.

Sensibility analysis: Meta-regression was suggested when at least contain ten studies in Handbook of Cochrane Review, but it is an iron-clad rule. Considering the inconclusive results based on high heterogeneity of the meta-lytic effects from eight studies, we tried to perform sensitivity analysis by meta-regression with covariates. Age, initial lactate levels and SOFA in HAT groups, and in-hospital mortality in control group indicated individual condition, severity of critical ill, as well as mixed factors of severity and care ability, respectively. We performed TSA to warrant the robustness of the result; thus, the threshold for statistical significance becomes more restrictive in TSA-evaluated meta-analysis and is now applied for the further evaluation of evidence-based critical care. We further performed leave-one-out sensitivity analysis.

Language: No restriction.

Country(ies) involved: Taiwan.
Keywords: hydrocortisone, ascorbic acid, thiamine, sepsis, septic shock, metaanalysis, trial sequential analysis.

\section{Contributions of each author:}

Author 1 - Chi-Hsin Chen - Database search and data extraction, critical analysis, interpretation of the data, and drafting of the manuscript.

Author 2 - Edward Pei-Chuan Huang Integration of the study and concept of meta-analysis.

Author 3 - James d'Etienne - Integration of the study and concept of meta-analysis.

Author 4 - Eric H. Chou - Integration of the study and concept of meta-analysis.

Author 5 - Yen-Hsing Liu - Provide informative suggestions for the preparation of the manuscript.

Author 6 - Yu-Sheng Huang - Provide informative suggestions for the preparation of the manuscript.

Author 7 - Jia-How Chang - Provide informative suggestions for the preparation of the manuscript.

Author 8 - Pei-Chun Lai - Double confirmation of the enrolled studies and data, concept of the meta-analysis, statistical analyses, grading of bias risk, and revision of the manuscript.

Author 9 - Yen-Ta Huang - Double confirmation of the enrolled studies and data, concept of the meta-analysis, statistical analyses, grading of bias risk, and revision of the manuscript. 